\title{
Stack Semantics of Type Theory
}

\author{
Thierry Coquand \\ Göteborgs Universitet
}

\author{
Bassel Mannaa \\ IT-Universitetet i København
}

\author{
Fabian Ruch \\ Göteborgs Universitet
}

April 2017

\begin{abstract}
We give a model of dependent type theory with one univalent universe and propositional truncation interpreting a type as a stack, generalizing the groupoid model of type theory. As an application, we show that countable choice cannot be proved in dependent type theory with one univalent universe and propositional truncation.
\end{abstract}

\section{Introduction}

The axiom of univalence [17, 16] can be seen as an extension to dependent type theory of the two axioms of extensionality for simple type theory as formulated by Church [2]. This extension is important since, using universe and dependent sums, we get a formal system in which we can represent arbitrary structures (which we can not do in simple type theory) with elegant formal properties. The goal of this paper is to contribute to the meta-theory of such systems by showing that Markov's principle and countable choice are not provable in dependent type theory extended with one univalent universe and propositional truncation. For simple type theory such independence results can be obtained by using sheaf semantics, respectively over Cantor space (for Markov's principle) and open unit interval $(0,1)$ (for countable choice). There are however problems with extending sheaf semantics to universes [10, 18]. In order to address these issues we use a suitable formulation of stack semantics, which, roughly speaking, replaces sets by groupoids. The notion of stack was introduced in algebraic geometry [8, 7] precisely in order to solve the same problems that one encounters when trying to extend sheaf semantics to type-theoretic universes. The compatibility condition for gluing local data is now formulated in terms of isomorphisms instead of strict equalities. In this sense, our model can also be seen as an extension of the groupoid model of type theory [9]. One needs to formulate some strict functoriality conditions on the stack gluing operation, which seem necessary to be able to get a model of the required equations of dependent type theory.

We see this work as a first step towards the proof of independence of countable choice from type theory with a hierarchy of univalent universes and propositional truncation, which we hope to obtain by an extension of our model to an $\infty$-stack version of cubical type theory [3.

The paper is organized as follows. We first present a slight variation of the groupoid model that we find convenient for expressing the stack semantics. We then explain how to represent propositional truncation in this setting, and how it can be used to formulate countable choice. We then notice that, even in a constructive meta-logic where countable choice fails, the axiom of countable choice does hold in this groupoid model. The groupoid model can be refined rather directly over a Kripke structure, and we present then our notion of stacks over a general topological space together with a proof that we get a model of dependent type theory with one univalent universe and propositional truncation. Instantiating our model to the case of Cantor space and open unit interval $(0,1)$ we obtain the results that Markov's principle and countable 


$$
\begin{aligned}
& \vdash() \quad \frac{\Gamma \vdash A}{\vdash \Gamma \cdot A} \\
& \frac{\vdash \Gamma}{\vdash 1: \Gamma \rightarrow \Gamma} \quad \frac{\vdash \tau: \Theta \rightarrow \Delta \quad \vdash \sigma: \Delta \rightarrow \Gamma}{\sigma \tau: \Theta \rightarrow \Gamma} \quad \frac{\Gamma \vdash A \quad \vdash \sigma: \Delta \rightarrow \Gamma}{\Delta \vdash A \sigma} \quad \frac{\Gamma \vdash A}{\Gamma \cdot A \vdash \mathbf{q}: A \mathbf{p}} \\
& \frac{\Gamma \vdash a: A \quad \vdash \sigma: \Delta \rightarrow \Gamma}{\Delta \vdash a \sigma: A \sigma} \\
& A 1=A \quad A(\sigma \tau)=(A \sigma) \tau \quad a 1=a \quad a(\sigma \tau)=(a \sigma) \tau \\
& \frac{\Gamma \vdash A}{\vdash \mathbf{p}: \Gamma . A \rightarrow \Gamma} \quad \frac{\Gamma \vdash A \quad \vdash \sigma: \Delta \rightarrow \Gamma \quad \Delta \vdash a: A \sigma}{\vdash(\sigma, a): \Delta \rightarrow \Gamma . A}
\end{aligned}
$$

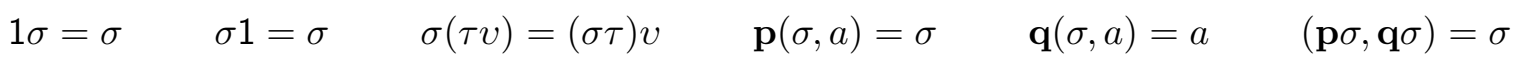

$$
\begin{aligned}
& \frac{\Gamma . A \vdash B}{\Gamma \vdash \Pi A B} \quad \frac{\Gamma \cdot A \vdash b: B}{\Gamma \vdash \lambda b: \Pi A B} \quad \frac{\Gamma \vdash f: \Pi A B \quad \Gamma \vdash a: A}{\Gamma \vdash \operatorname{app}(f, a): B[a]} \\
& \operatorname{app}(\lambda b, a)=b[a] \quad \lambda \operatorname{app}(f \mathbf{p}, \mathbf{q})=f
\end{aligned}
$$

Figure 1: Type theory

choice cannot be proved in dependent type theory with one univalent universe and propositional truncation.

\section{Type theory}

As in [1], we will use a generalized algebraic presentation of type theory that is name-free and has explicit substitutions. For instance, if we write $A \rightarrow B$ for $\Pi A(B \mathbf{p})$ then we have $\Gamma \vdash \lambda \mathbf{q}: A \rightarrow A$ since $\Gamma . A \vdash \mathbf{q}: A \mathbf{p}$. The advantage of using such a presentation is that it makes it easier to check the correctness of the model: Building such a model is reduced to defining operations such that certain equations hold. The main rules are presented in figures 1, 2, 3 and 4, We omit equivalence, congruence and substitution rules. The conversion rules assume appropriate typing premises.

We write $[a]$ for the substitution $(1, a)$ and $[a, b]$ for $([a], b)$.

\section{Groupoid model}

In this section, we review the groupoid model of [9], with a slightly different presentation inspired from [13]. We work in a set theory with a Grothendieck universe $\mathscr{U}$ (or a suitable constructive version of it if we work in a constructive set theory such as CZF [5]).

A groupoid is given by a set $\Gamma$ of objects and for each $\rho, \rho^{\prime} \in \Gamma$ a set $\Gamma\left(\rho, \rho^{\prime}\right)$ of paths/isomorphisms along with a composition operation $\alpha \cdot \alpha^{\prime}$ in $\Gamma\left(\rho, \rho^{\prime \prime}\right)$ for $\alpha$ in $\Gamma\left(\rho, \rho^{\prime}\right)$ and $\alpha^{\prime}$ in $\Gamma\left(\rho^{\prime}, \rho^{\prime \prime}\right)$ and a unit element $1_{\rho}$ in $\Gamma(\rho, \rho)$ and an inverse operation $\alpha^{-1}$ in $\Gamma\left(\rho^{\prime}, \rho\right)$ satisfying the usual unit, inverse and associativity laws. We may write $\alpha: \rho \cong \rho^{\prime}$ for $\alpha$ in $\Gamma\left(\rho, \rho^{\prime}\right)$. 


$$
\frac{\Gamma \vdash A \text { small } \quad \Gamma . A \vdash B \text { small }}{\Gamma \vdash \Pi A B \text { small }} \quad \frac{\Gamma . A \vdash B \text { discrete }}{\Gamma \vdash \Pi A B \text { discrete }}
$$

$$
\begin{array}{cc}
\frac{\Gamma \vdash A \text { small discrete }}{\Gamma \vdash|A|: \mathrm{U}} & \frac{\Gamma \vdash a: \mathrm{U}}{\Gamma \vdash \text { El } a \text { small discrete }} \\
\mathrm{El}|A|=A & |\mathrm{El} a|=a \\
\frac{\Gamma \vdash A \text { small }}{\Gamma \vdash A} & \frac{\Gamma \vdash A \text { discrete }}{\Gamma \vdash A}
\end{array}
$$

Figure 2: Universe in type theory

$$
\begin{aligned}
& \frac{\Gamma \vdash A \quad \Gamma \vdash a: A \quad \Gamma \vdash b: A}{\Gamma \vdash \text { Path } A a b \text { discrete }} \quad \frac{\Gamma \vdash A \text { small } \quad \Gamma \vdash a: A \quad \Gamma \vdash b: A}{\Gamma \vdash \text { Path } A a b \text { small }} \\
& \Gamma \vdash A \quad \Gamma . A . A \text { p.Path } A \text { pp qp q } \vdash C
\end{aligned}
$$

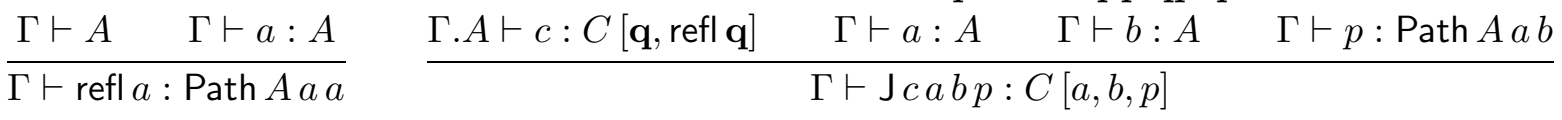

$$
\begin{aligned}
& \mathrm{J} c a a(\operatorname{refl} a)=c[a]
\end{aligned}
$$

Figure 3: Equality in type theory

$$
\begin{aligned}
& \frac{\Gamma . A \vdash B}{\Gamma \vdash \Sigma A B} \quad \frac{\Gamma \vdash a: A \quad \Gamma \vdash b: B[a]}{\Gamma \vdash(a, b): \Sigma A B} \quad \frac{\Gamma \vdash p: \Sigma A B}{\Gamma \vdash p .1: A} \quad \frac{\Gamma \vdash p: \Sigma A B}{\Gamma \vdash p .2: B[p .1]} \\
& \begin{array}{lll}
(a, b) .1=a & (a, b) .2=b \quad(p .1, p .2)=p
\end{array} \\
& \Gamma \vdash \mathrm{N} \text { small discrete } \quad \Gamma \vdash 0: \mathrm{N} \quad \frac{\Gamma \vdash n: \mathrm{N}}{\Gamma \vdash \operatorname{suc} n: \mathrm{N}} \\
& \frac{\Gamma . \mathrm{N} \vdash C \quad \Gamma \vdash c: C[0] \quad \Gamma . \mathrm{N} . C \vdash d: C[\operatorname{suc} \mathbf{q}] \mathbf{p} \quad \Gamma \vdash n: \mathbf{N}}{\Gamma \vdash \operatorname{rec} c d n: C[n]} \\
& \operatorname{rec} c d 0=c \quad \operatorname{rec} c d(\operatorname{suc} n)=d[n, \operatorname{rec} c d n] \\
& \Gamma \vdash \mathrm{N}_{2} \text { small discrete } \quad \Gamma \vdash 0: \mathrm{N}_{2} \quad \Gamma \vdash 1: \mathrm{N}_{2} \\
& \frac{\Gamma . \mathrm{N}_{2} \vdash C \quad \Gamma \vdash c: C[0] \quad \Gamma \vdash d: C[1] \quad \Gamma \vdash b: \mathrm{N}_{2}}{\Gamma \vdash \operatorname{rec}_{2} c d b: C[b]} \\
& \operatorname{rec}_{2} c d 0=c \quad \quad \operatorname{rec}_{2} c d 1=d
\end{aligned}
$$

Figure 4: Dependent sum, natural numbers and Booleans in type theory 
A map $\sigma: \Delta \rightarrow \Gamma$ between two groupoids $\Delta$ and $\Gamma$ is given by a set-theoretic map $\sigma \nu$ in $\Gamma$ for $\nu$ in $\Delta$ and a map $\sigma \beta$ in $\Gamma\left(\sigma \nu, \sigma \nu^{\prime}\right)$ for $\beta$ in $\Delta\left(\nu, \nu^{\prime}\right)$ which commutes with unit, inverse and composition.

A family $A$ of groupoids indexed over a groupoid $\Gamma$, written $\Gamma \vdash A$, is given by a family of sets $A \rho$ for each $\rho$ in $\Gamma$ and sets $A \alpha\left(u, u^{\prime}\right)$ for each $\alpha$ in $\Gamma\left(\rho, \rho^{\prime}\right)$ and $u \in A \rho$ and $u^{\prime} \in A \rho^{\prime}$. We may write $\omega: u \cong_{\alpha} u^{\prime}$ for $\omega$ element of $A \alpha\left(u, u^{\prime}\right)$ and we may omit the subscript $\alpha$ if it is clear from the context. We also have unit $1_{u}: u \cong_{1_{\rho}} u$ and inverse $\omega^{-1}: u^{\prime} \cong_{\alpha^{-1}} u$ and composition $\omega \cdot \omega^{\prime}: u \cong{ }_{\alpha \cdot \alpha^{\prime}} u^{\prime \prime}$ also satisfying the unit, inverse and associativity laws. We furthermore should have a path lifting structure, which is given by two operations $u \alpha$ in $A \rho^{\prime}$ and $u \uparrow \alpha: u \cong_{\alpha} u \alpha$ for $u$ in $A \rho$ and $\alpha: \rho \cong \rho^{\prime}$ satisfying the laws

$$
u 1_{\rho}=u \quad(u \alpha) \alpha^{\prime}=u\left(\alpha \cdot \alpha^{\prime}\right) \quad u \uparrow 1_{\rho}=1_{u} \quad(u \uparrow \alpha) \cdot\left(u \alpha \uparrow \alpha^{\prime}\right)=u \uparrow\left(\alpha \cdot \alpha^{\prime}\right)
$$

We see that $u \uparrow \alpha$ "lifts" the path $\alpha: \rho \cong \rho^{\prime}$ given an initial point $u$ in $A \rho$.

Each $A \rho$ has a canonical groupoid structure, defining $A \rho\left(u, u^{\prime}\right)$ to be $A 1_{\rho}\left(u, u^{\prime}\right)$. If $\alpha: \rho \cong \rho^{\prime}$ we can define a groupoid map $A \rho \rightarrow A \rho^{\prime}$ using the lifting operation. We thereby recover the groupoid model as defined in [9].

If $\sigma: \Delta \rightarrow \Gamma$ and $\Gamma \vdash A$ we define $\Delta \vdash A \sigma$ by composition: $(A \sigma) \nu$ is $A(\sigma \nu)$ and $(A \sigma) \beta\left(v, v^{\prime}\right)$ is $A(\sigma \beta)\left(v, v^{\prime}\right)$.

A section $\Gamma \vdash a: A$ is given by a family of objects $a \rho$ in $A \rho$ together with a family of paths $a \alpha: a \rho \cong_{\alpha} a \rho^{\prime}$ satisfying the laws $a 1_{\rho}=1_{a \rho}$ and $a\left(\alpha \cdot \alpha^{\prime}\right)=a \alpha \cdot a \alpha^{\prime}$.

If $\Gamma \vdash A$, we define a new groupoid $\Gamma$.A: An object $(\rho, u)$ in $\Gamma$.A is a pair with $\rho$ in $\Gamma$ and $u$ in $A \rho$ and a path $(\alpha, \omega):(\rho, u) \cong\left(\rho^{\prime}, u^{\prime}\right)$ is a pair $\alpha: \rho \cong \rho^{\prime}$ and $\omega: u \cong{ }_{\alpha} u^{\prime}$. We then have $\mathbf{p}: \Gamma . A \rightarrow \Gamma$ defined by $\mathbf{p}(\rho, u)=\rho$ and $\mathbf{p}(\alpha, \omega)=\alpha$ and the section $\Gamma \cdot A \vdash \mathbf{q}: A \mathbf{p}$ defined by $\mathbf{q}(\rho, u)=u$ and $\mathbf{q}(\alpha, \omega)=\omega$.

We say that a family $\Gamma \vdash A$ is small if each set $A \rho$ and $A \alpha\left(u, u^{\prime}\right)$ is in the given Grothendieck universe $\mathcal{U}$. We say that this family is discrete if the lifting is uniquely determined: Given $u$ in $A \rho$ and $\alpha: \rho \cong \rho^{\prime}$ there is a unique $u^{\prime}$ in $A \rho^{\prime}$ such that $A \alpha\left(u, u^{\prime}\right)$ is inhabited and this set is a singleton in this case. This notion of discrete family can be characterized in terms of the common definition of discrete groupoid, which says that a groupoid is discrete if the only paths are units.

Lemma 1. $\Gamma \vdash A$ is discrete if and only if each groupoid $A \rho, \rho \in \Gamma$, is discrete.

Proof. Assume $\Gamma \vdash A$ to be a discrete family and let $\omega \in A 1_{\rho}\left(u, u^{\prime}\right)$ be an arbitrary path. We immediately have $u^{\prime}=u$ and $\omega=1_{u}$ by discreteness of $\Gamma \vdash A$ and $1_{u} \in A 1_{\rho}(u, u)$.

For each $\rho \in \Gamma$, assume $A \rho$ to be a discrete groupoid and let $\omega^{\prime} \in A \alpha\left(u, u^{\prime}\right), \omega^{\prime \prime} \in A \alpha\left(u, u^{\prime \prime}\right)$ be two arbitrary paths over some $\alpha \in \Gamma\left(\rho, \rho^{\prime}\right)$. Then, $\omega^{\prime \prime}$ can be expressed as the composite of $\omega^{\prime}$ and $\omega^{\prime-1} \cdot \omega^{\prime \prime} \in A 1_{\rho^{\prime}}\left(u^{\prime}, u^{\prime \prime}\right)$. The discreteness of $A \rho^{\prime}$ forces $\omega^{-1} \cdot \omega^{\prime \prime}$ to be a unit path so that $u^{\prime \prime}=u^{\prime}$ and $\omega^{\prime \prime}=\omega^{\prime}$.

We define $\mathrm{U}$ to be the following groupoid: An object $X$ in $\mathrm{U}$ is exactly an element of the given Grothendieck universe $\mathcal{U}$, and an element of $\mathrm{U}\left(X, X^{\prime}\right)$ is a bijection between $X$ and $X^{\prime}$. We can then define the small and discrete family $\mathrm{U} \vdash$ El by taking El $X$ to be the set $X$ and $u \cong_{\alpha} u^{\prime}$ to be the subsingleton set $\left\{0 \mid u^{\prime}=\alpha u\right\}$, that is $u \cong_{\alpha} u^{\prime}$ is inhabited and is the singleton $\{0\}$ exactly when $u^{\prime}=\alpha u$.

Proposition 1. The family $\mathrm{U} \vdash \mathrm{El}$ is a universal small and discrete family: If $\Gamma \vdash A$ is small and discrete, then there exists a unique map $|A|: \Gamma \rightarrow \mathrm{U}$ such that $\mathrm{El}|A|=A$ (with strict equality). 
For $\Gamma \vdash A$ and $\Gamma$. $A \vdash B$ we define $\Gamma \vdash \Pi A B$ by taking $(\Pi A B) \rho$ to be the set of functions $c u$ in $B(\rho, u)$ and $c \omega$ in $B\left(1_{\rho}, \omega\right)\left(c u, c u^{\prime}\right)$ commuting with unit and composition, and ( $\left.\Pi A B\right) \alpha\left(c, c^{\prime}\right)$ to be the set of functions $\gamma \omega: c u \cong(\alpha, \omega) c^{\prime} u^{\prime}$ such that $\left(\gamma \omega_{0}\right) \cdot\left(c^{\prime} \beta^{\prime}\right)=(c \beta) \cdot\left(\gamma \omega_{1}\right)$ if $\beta: u_{0} \cong_{\rho} u_{1}$ and $\beta^{\prime}: u_{0}^{\prime} \cong \rho_{\rho^{\prime}} u_{1}^{\prime}$ and $\omega_{0}: u_{0} \cong_{\alpha} u_{0}^{\prime}$ and $\omega_{1}: u_{1} \cong_{\alpha} u_{1}^{\prime}$. There is then [9, 13] a canonical way to define a composition operation (we need the path lifting structure for $\Gamma \vdash A$ ) and path lifting structure for $\Gamma \vdash \Pi A B$.

Proposition 2. If $\Gamma . A \vdash B$ is discrete, then so is $\Gamma \vdash \Pi A B$.

Proof. In order to show that $\Gamma \vdash \Pi A B$ is a discrete family, it suffices to show that $(\Pi A B) \rho$ is a discrete groupoid for each $\rho \in \Gamma$. Assume $\Gamma$. $A \vdash B$ to be a discrete family and let $\gamma \in$ $(\Pi A B) 1_{\rho}\left(c, c^{\prime}\right)$ be an arbitrary path. In particular, $B u$ is a discrete groupoid forcing $\gamma 1_{u} \in$ $B 1_{u}\left(c u, c^{\prime} u\right)$ to be a unit path for each $u \in A \rho$ so that $c^{\prime} u=c u$ for all $u \in A \rho$. The discreteness of $\Gamma$. $A \vdash B$ also forces $c^{\prime} \omega=c \omega$ and $\gamma \omega=1_{c} \omega$ in $B \omega\left(c u^{\prime}, c u^{\prime \prime}\right)$ for all $\omega \in A 1_{\rho}\left(u^{\prime}, u^{\prime \prime}\right)$, which concludes $c^{\prime}=c$ and $\gamma=1_{c}$.

If $\Gamma \vdash A$ and $\Gamma \vdash a_{0}: A$ and $\Gamma \vdash a_{1}: A$ we define the discrete family $\Gamma \vdash$ Path $A a_{0} a_{1}$. We take (Path $\left.A a_{0} a_{1}\right) \rho$ for $\rho \in \Gamma$ to be the set $A 1_{\rho}\left(a_{0} \rho, a_{1} \rho\right.$ ) and (Path $\left.A a_{0} a_{1}\right) \alpha\left(\omega, \omega^{\prime}\right)$ for $\alpha: \rho \cong \rho^{\prime}$ to be the subsingleton $\left\{0 \mid \omega \cdot a_{1} \alpha=a_{0} \alpha \cdot \omega^{\prime}\right\}$.

It is then possible [9, 13] to check that this defines a model of type theory as presented by the rules of figures 1, 2, 3 and 4.

\subsection{Propositional truncation}

We say that a groupoid is a proposition if and only if there exists exactly one path between two objects. So $\Gamma$ is a proposition if and only if each set $\Gamma\left(\rho, \rho^{\prime}\right)$ is a singleton. More generally, we say that a family $\Gamma \vdash A$ is a proposition if each set $A \alpha\left(u, u^{\prime}\right)$ is a singleton.

Lemma 2. $\Gamma \vdash A$ is a proposition if and only if each groupoid $A \rho, \rho \in \Gamma$, is a proposition.

Proof. It is clear that each $A \rho$ is a proposition if the whole family $\Gamma \vdash A$ is a proposition.

Assume now each $A \rho$ to be a proposition and let $\alpha \in \Gamma\left(\rho, \rho^{\prime}\right)$ as well as $u \in A \rho, u^{\prime} \in A \rho^{\prime}$. Then, the set $A \alpha\left(u, u^{\prime}\right)$ is inhabited by the composite $(u \uparrow \alpha) \cdot p_{u \alpha, u^{\prime}}$ of the lifting of $u$ over $\alpha$ with the unique path between $u \alpha$ and $u^{\prime}$ in $A \rho^{\prime}$. Furthermore, for any two paths $\omega, \omega^{\prime} \in A \alpha\left(u, u^{\prime}\right)$ the composite $\omega^{-1} \cdot \omega^{\prime}$ is forced to be the unit path at $u^{\prime}$ so that $\omega^{\prime}=\omega \cdot \omega^{-1} \cdot \omega^{\prime}=\omega$.

We define as usual (where names are used for readability)

$$
\text { isProp } A=\Pi\left(x_{0} x_{1}: A\right) \text { Path } A x_{0} x_{1}
$$

Proposition 3. If $\Gamma \vdash A$, then there exists a section $\Gamma \vdash p$ : isProp $A$ if and only if each groupoid $A \rho, \rho$ in $\Gamma$, is a proposition.

Proof. It is enough to show that there exists a family of paths $p_{\rho, u, u^{\prime}} \in A 1_{\rho}\left(u, u^{\prime}\right), u, u^{\prime} \in A \rho$, $\rho \in \Gamma$, satisfying $p_{\rho, u, u^{\prime}} \cdot \omega^{\prime}=\omega \cdot p_{\rho^{\prime}, v, v^{\prime}}$ for all $\omega \in A \alpha(u, v)$ and $\omega^{\prime} \in A \alpha\left(u^{\prime}, v^{\prime}\right), u, u^{\prime} \in A \rho$, $v, v^{\prime} \in A \rho^{\prime}, \alpha \in \Gamma\left(\rho, \rho^{\prime}\right), \rho, \rho^{\prime} \in \Gamma$, if and only if each groupoid $A \rho, \rho \in \Gamma$, is a proposition.

Assume such a family $p$ and let $\rho \in \Gamma, u, u^{\prime} \in A \rho$, then $A 1_{\rho}\left(u, u^{\prime}\right)$ is inhabited by the composite $p_{\rho, u, u^{\prime}} \cdot\left(p_{\rho, u^{\prime}, u^{\prime}}\right)^{-1}$ and, moreover, any other path $\omega \in A 1_{\rho}\left(u, u^{\prime}\right)$ satisfies $p_{\rho, u, u^{\prime}} \cdot 1_{u^{\prime}}=$ $\omega \cdot p_{\rho, u^{\prime}, u^{\prime}}$ so that $A 1_{\rho}\left(u, u^{\prime}\right)$ is indeed a singleton.

In the opposite direction, we can actually assume the whole family $\Gamma \vdash A$ to be a proposition. Then, defining $p_{\rho, u, u^{\prime}}$ to be the unique path from $u$ to $u^{\prime}$ satisfies $p_{\rho, u, u^{\prime}} \cdot \omega^{\prime}=\omega \cdot p_{\rho^{\prime}, v, v^{\prime}}$ because there exists exactly one path from $u$ to $v^{\prime}$ over $\alpha$. 
For $\Gamma \vdash A$ we define $\Gamma \vdash\|A\|$ as follows. For each $\rho$ in $\Gamma$ we take $\|A\| \rho=A \rho$, and for each $\alpha$ in $\Gamma\left(\rho, \rho^{\prime}\right), u$ in $A \rho$ and $u^{\prime}$ in $A \rho^{\prime}$ we take $\|A\| \alpha\left(u, u^{\prime}\right)$ to be a fixed singleton $\{0\}$. We then have sections of $\Gamma \vdash$ isProp $\|A\|$ and $\Gamma \vdash A \rightarrow\|A\|$, and given sections of $\Gamma \vdash$ isProp $B$ and $\Gamma \vdash A \rightarrow B$ there is a section of $\Gamma \vdash\|A\| \rightarrow B$. In this way, we get a model of the propositional truncation operation.

\subsection{Countable choice}

The statement of countable choice can be formulated as the type 16]

$$
\mathrm{CC}=\Pi(A: \mathrm{N} \rightarrow \mathrm{U})(\Pi(n: \mathrm{N})\|\mathrm{El}(A n)\|) \rightarrow\|\Pi(n: \mathrm{N}) \mathrm{El}(A n)\|
$$

Notice that we can develop the groupoid model in a constructive meta-theory where countable choice may or may not hold.

Theorem 1. The statement CC is valid in the groupoid model (even if countable choice does not hold in the meta-theory).

Proof. It is enough to define $c A f=f$ and $c \alpha \omega=0$ to get ()$\vdash c$ : CC.

\section{Stack model}

\subsection{Groupoid-valued presheaf model}

We suppose given a poset with elements $U, V, W, X, \ldots$ The groupoid model extends directly as a groupoid-valued presheaf model over this poset. A context is now a family of groupoids $\Gamma(U)$ indexed by elements of the given poset such that objects $\rho$ and paths $\alpha$ in $\Gamma(U)$ can be restricted to $\rho \mid V$ and $\alpha \mid V$ in $\Gamma(V)$ if $V \subseteq U$ such that the restriction operation defines a groupoid map $\Gamma(U) \rightarrow \Gamma(V)$ which is the identity map for $V=U$ and the composite of $\Gamma(X) \rightarrow \Gamma(V)$ and $\Gamma(U) \rightarrow \Gamma(X)$ for $V \subseteq X \subseteq U$.

For a given context $\Gamma$, we define then what is a family $\Gamma \vdash A$. It is given by a family of sets $A \rho$ for each $U$ and $\rho$ in $\Gamma(U)$ together with a restriction $u \mid V$ in $A(\rho \mid V)$ for $u$ in $A \rho$ satisfying $u \mid U=u$ and $(u \mid X)|V=u| V$, as well as a family of sets $A \alpha\left(u, u^{\prime}\right)$ for each $\alpha: \rho \cong \rho^{\prime}$ in $\Gamma(U)$, $u$ in $A \rho$ and $u^{\prime}$ in $A \rho^{\prime}$ together with a restriction $\omega \mid V$ in $A(\alpha \mid V)\left(u\left|V, u^{\prime}\right| V\right)$ for $\omega$ in $A \alpha\left(u, u^{\prime}\right)$ satisfying $\omega \mid U=\omega$ and $(\omega \mid X)|V=\omega| V$. In particular, we require the restriction operation on the sets $A \alpha\left(u, u^{\prime}\right)$ to commute with unit and composition. Such a family is called small if the sets $A \rho$ and $A \alpha\left(u, u^{\prime}\right)$ are elements in the Grothendieck universe $\mathcal{U}$, and it is called a proposition if the canonical groupoid structure on each $A \rho$ defines a proposition, or, equivalently, if each set $A \alpha\left(u, u^{\prime}\right)$ is a singleton. Furthermore, we should have a lifting operation $u \uparrow \alpha$ with the law $(u \uparrow \alpha) \mid V=(u \mid V) \uparrow(\alpha \mid V)$. A family is called discrete if the liftings $u \uparrow \alpha$ are uniquely determined: Given $U$ and $\rho$ in $\Gamma(U)$ and given $u$ in $A \rho$ and $\alpha: \rho \cong \rho^{\prime}$ there is a unique $u^{\prime}$ in $A \rho^{\prime}$ such that $A \alpha\left(u, u^{\prime}\right)$ is inhabited, and this set is a singleton in this case.

We can extend the groupoid model to this setting.

An element $c$ of $(\Pi A B) \rho$ for $\rho$ in $\Gamma(U)$ is a function $c u$ in $B(\rho \mid V, u)$ for $V \subseteq U$ and $u$ in $A(\rho \mid V)$ and $c \omega$ in $B\left(1_{\rho \mid V}, \omega\right)\left(c u, c u^{\prime}\right)$ for $\omega$ in $A 1_{\rho \mid V}\left(u, u^{\prime}\right)$ commuting with unit and composition such that $(c a) \mid W=c(a \mid W)$ and $(c \omega) \mid W=c(\omega \mid W)$ if $W \subseteq V \subseteq U$.

An element in $(\Sigma A B) \rho$ for $\rho \in \Gamma(U)$ is a pair $(a, b)$ where $a \in A \rho$ and $b \in B(\rho, a)$ with restrictions $(a, b) \mid V=(a|V, b| V)$. Paths in $(\Sigma A B) \alpha\left((a, b),\left(a^{\prime}, b^{\prime}\right)\right)$, where $\alpha: \rho \cong \rho^{\prime}$ are pairs $(\omega, \mu)$ where $\omega: a \cong_{\alpha} a^{\prime}$ and $\mu: b \cong_{(\alpha, \omega)} b^{\prime}$ with restrictions $(\omega, \mu) \mid V=(\omega|V, \mu| V)$.

Given sections $a_{0}$ and $a_{1}$ of $A$, an element in (Path $\left.A a_{0} a_{1}\right) \rho$ for $\rho \in \Gamma(U)$ is a path $\omega: a_{0} \rho \cong$ $a_{1} \rho$ with restrictions as in $A$. For every element $\omega$ and path $\alpha: \rho \cong \rho^{\prime}$ there is a unique path from $\omega$ over $\alpha$ going to $\left(a_{0} \alpha\right)^{-1} \cdot \omega \cdot a_{1} \alpha: a_{0} \rho^{\prime} \cong a_{1} \rho^{\prime}$. 


\subsection{Stack structure}

We assume given a topological space with a notion of basic open closed under nonempty intersection and a notion of covering of a given basic open by a family of basic opens. We consider only coverings $\left(U_{i}\right)_{i \in I}$ of some basic open $U$ where the set of indices $I$ is small. To simplify the presentation we assume that each basic open set is nonempty. We write $U_{i j}$ for $U_{i} \cap U_{j}$ and $U_{i j k}$ for $U_{i} \cap U_{j} \cap U_{k}$ when they are nonempty.

Since basic opens form a poset, we can consider the notion of type family over this poset as defined in the previous subsection.

In the following we will define what is a stack structure on a type family.

We recall that a sheaf $F$ is given by a presheaf, i.e. a family of sets $F(U)$ with restriction maps $u \mid V$ in $F(V)$ for $V \subseteq U$ such that $u \mid U=u$ and $(u \mid V)|W=u| W$ if $W \subseteq V \subseteq U$, which satisfies the condition that if we have a covering $\left(U_{i}\right)_{i \in I}$ of $U$ and a family of compatible elements $u_{i}$ in $F\left(U_{i}\right)$ (i.e. $\left.u_{i}\left|U_{i j}=u_{j}\right| U_{i j}\right)$ then there exists a unique $u$ in $F(U)$ such that $u \mid U_{i}=u_{i}$ for all $i$.

A type family $\Gamma \vdash A$ is called a prestack if it satisfies the following sheaf condition on paths: If $\alpha: \rho \cong \rho^{\prime}$ is in $\Gamma(U), u$ and $u^{\prime}$ are in $A \rho$ and $A \rho^{\prime}$ respectively and we have a family of paths $\omega_{i}: u\left|U_{i} \cong_{\alpha \mid U_{i}} u^{\prime}\right| U_{i}$ which is compatible (that is $\omega_{i}\left|U_{i j}=\omega_{j}\right| U_{i j}$ ), then we have a unique path $\omega: u \cong_{\alpha} u^{\prime}$ such that $\omega \mid U_{i}=\omega_{i}$ for all $i$.

For each basic open $U$ and $\rho$ in $\Gamma(U)$ we define what is the set of descent data $D(A) \rho$. A descent datum is given by a covering $\left(U_{i}\right)_{i \in I}$ of $U$ and a family of objects $u_{i} \in A\left(\rho \mid U_{i}\right)$ with paths $\varphi_{i j}: u_{i}\left|U_{i j} \cong_{\rho \mid U_{i j}} u_{j}\right| U_{i j}$, when $U_{i}$ meets $U_{j}$, satisfying the cocycle condition: 1$]$

$$
\varphi_{i i}=1_{u_{i}} \quad \varphi_{i j}\left|U_{i j k} \cdot \varphi_{j k}\right| U_{i j k}=\varphi_{i k} \mid U_{i j k}
$$

This forms a set since the index set is restricted to be small (otherwise this might be a proper class in general).

If $d=\left(u_{i}, \varphi_{i j}\right)$ is an element of $D(A) \rho$ and $V \subseteq U$ we define its restriction $d \mid V$, element of $D(A) \rho \mid V$, which is the family $\left(u_{i}\left|V \cap U_{i}, \varphi_{i j}\right| V \cap U_{i j}\right)$ restricted to indices $i$ such that $V$ meets $U_{i}$. A gluing operation glue $d=\left(u, \varphi_{i}\right)$ gives an element $u$ in $A \rho$ together with paths $\varphi_{i}: u \mid U_{i} \cong u_{i}$ such that $\varphi_{i}\left|U_{i j} \cdot \varphi_{i j}=\varphi_{j}\right| U_{i j}$ and satisfies the law (glue $\left.d\right) \mid V=\operatorname{glue}(d \mid V)$, that is glue $(d \mid V$ ) should be $\left(u\left|V, \varphi_{i}\right| V \cap U_{i}\right)$ where we restrict the family to indices $i$ such that $V$ meets $U_{i}$. This functoriality property will be crucial for checking that we do get a model of type theory with dependent product.

A stack structure on a prestack $\Gamma \vdash A$ is given by a gluing operation 2 .

Consider a prestack $\Gamma \vdash A$ and descent datum $d=\left(u_{i}, \varphi_{i j}\right) \in D(A) \rho$ with glue $d=\left(u, \varphi_{i}\right)$ as above. Let $v \in A \rho$ with paths $\vartheta_{i}: v \mid U_{i} \cong u_{i}$ satisfying $\vartheta_{i}\left|U_{i j} \cdot \varphi_{i j}=\vartheta_{j}\right| U_{i j}$. We remark that while it is not necessarily true that $u=v$, the prestack condition implies that we have a path $v \cong u$.

Note that while it is sufficient to define the notion of sheaf as a property because of the uniqueness part of the sheaf condition, it is crucial that our notion of stack is in general a structure, i.e. given with an explicit operation fixing a particular choice of glue.

A stack is not the same as a groupoid object in the sheaf topos. A prime example of a stack whose presheaf of objects is not a sheaf is the universe of sheaves: If we define $F(U)$ to be the collection of small sheaves over $U$ then there is a natural restriction operation $F(U) \rightarrow F(V)$ for $V \subseteq U$, and one can check that the gluing of a compatible family of elements is not unique up to strict equality in general (but it is unique up to isomorphism). Notice that if we try to define the stack structure using global choice as in [8,3.3.1, page 28] then the functoriality condition

\footnotetext{
${ }^{1}$ The first condition is not logically necessary.

${ }^{2}$ Notice that we shall not require the context $\Gamma$ to be a prestack or have a stack structure.
} 
(glue $d) \mid V=\operatorname{glue}(d \mid V)$ will not hold. There is however a more canonical definition of gluing which satisfies this condition, which will provide the interpretation of a univalent universe.

There is also a simple example of a prestack that is not a stack but whose presheaf of objects is a sheaf. Consider the topological space given by basic opens $U_{1}, U_{2}, U_{12}$ with $U_{1} \wedge U_{2}=U_{12}$ and the groupoid-valued presheaf $G$ given by the propositions on the sets $G(1)=\emptyset, G\left(U_{1}\right)=\left\{x_{1}\right\}$, $G\left(U_{2}\right)=\left\{x_{2}\right\}$ and $G\left(U_{12}\right)=\left\{x_{1}, x_{2}\right\}$. There are no matching families of objects or morphisms in $G$ so that both the presheaf of objects and the presheaf of morphisms trivially satisfy the sheaf property. However, the descent datum given by $d_{1}=x_{1}, d_{2}=x_{2}$ and $d_{12}$ the unique path between $x_{1} \mid U_{12}$ and $x_{2} \mid U_{12}$ cannot have a glue because $G(1)$ is empty.

Taking as objects the subset $D(A)(\rho, C) \subseteq D(A) \rho$ of descent data on a covering $C=\left(U_{i}\right)_{i \in I}$ of $U$ and a path between two descent data $\left(u_{i}, \varphi_{i j}\right)$ and $\left(v_{i}, \psi_{i j}\right)$ to be a family of paths $\omega_{i}$ : $u_{i} \cong v_{i}$ satisfying $\omega_{i} \cdot \psi_{i j}=\varphi_{i j} \cdot \omega_{j}$ has a natural groupoid structure. Moreover, the canonical restriction from $A \rho$ to $D(A) \rho$ extends to a functor from the canonical groupoid structure on $A \rho$ to $D(A)(\rho, C)$. The prestack condition for $A$ then says that this functor is fully faithful and a gluing operation witnesses that it is essentially surjective. If $A$ is a stack, then the canonical functor $A \rho \rightarrow D(A)(\rho, C)$ is an equivalence of groupoids.

\subsection{Dependent product}

The collection of types with a stack structure is closed under dependent product.

Theorem 2. If $\Gamma . A \vdash B$ has a stack structure then $\Gamma \vdash \Pi A B$ has a stack structure.

Proof. Let $\left(u_{i}, \varphi_{i j}\right) \in D(\Pi A B) \rho$ be a descent datum on a covering $\left(U_{i}\right)_{i \in I}$ of $U$. We construct a glue $\left(u, \varphi_{i}\right)$ that commutes with restriction.

Given $x, x^{\prime} \in A(\rho \mid V)$ and $\nu: x \cong x^{\prime}$ on $V \subseteq U$, we construct $\left(u x, \varphi_{i} x\right)$ as the glue of $d_{x}=$ $\left(u_{i} x, \varphi_{i j} x\right)$ and $u \nu: u x \cong_{\nu} u x^{\prime}$ as the unique path matching $u x \cong u_{i} x \cong_{\nu \mid V \cap U_{i}} u_{i} x^{\prime} \cong u x^{\prime}$ given by the composite of $\varphi_{i} x, u_{i} \nu$ and the inverse of $\varphi_{i} x^{\prime}$ on $V \cap U_{i}$. If in particular $V \subseteq U_{i}$, then this completely determines $\varphi_{i}: u \mid U_{i} \cong u_{i}$. The uniqueness of $u \nu$ is needed to show that $u$ respects units and composites as well as restriction of paths. For $u$ to also respect restriction of objects we need the fact that $\left(\right.$ glue $\left.d_{x}\right) \mid W=$ glue $d_{x} \mid W=$ glue $d_{x \mid W}$ for $W \subseteq V$.

Let $\omega_{i}: u\left|U_{i} \cong{ }_{\alpha \mid U_{i}} u^{\prime}\right| U_{i}$ be a matching family of paths. We show that there is a unique glue $\omega: u \cong_{\alpha} u^{\prime}$. It is uniquely determined by the glues $\omega \nu: u x \cong_{(\alpha \mid V, \nu)} u^{\prime} x^{\prime}$ of $\omega_{i} \nu$ : $u x \cong_{\left(\alpha\left|V \cap U_{i}, \nu\right| V \cap U_{i}\right)} u^{\prime} x^{\prime}$ for $\nu: x \cong_{\alpha \mid V} x^{\prime}$. In particular, $\omega \nu=\omega_{i} \nu$ if $V \subseteq U_{i}$. Again, the uniqueness of $\omega \nu$ lets us show that $\omega$ respects composites and restrictions.

\subsection{Universe of sheaves}

We define $\mathrm{U}(V)$ to be the collection of all small sheaves over $V$. There is a natural restriction operation $\mathrm{U}(V) \rightarrow \mathrm{U}(W)$ if $W \subseteq V$.

Theorem 3. U has a stack structure.

Proof. Let $F_{i} \in \mathrm{U}\left(U_{i}\right)$ with $\varphi_{i j}: F_{i}\left|U_{i j} \cong F_{j}\right| U_{i j}$ be a descent datum on a cover $\left(U_{i}\right)_{i \in I}$ of $U$. We construct a glue $F \in \mathrm{U}(U)$ and $\varphi_{i}: F \mid U_{i} \cong F_{i}$. We define $F(V)$ for $V \subseteq U$ as the set of families $\left(x_{i}\right)_{i}$ where $x_{i} \in F_{i}\left(V \cap U_{i}\right)$ and $\varphi_{i j}\left(x_{i}\right)=x_{j}$. Furthermore, we define $F(V) \rightarrow F(W)$ for $W \subseteq V$ component-wise by the restriction $F_{i}\left(V \cap U_{i}\right) \rightarrow F_{i}\left(W \cap U_{i}\right)$ and $\varphi_{i}$ by the projection to the $i$-th component. For $\varphi_{i}$ to be an isomorphism we need the fact $\varphi_{i i}=1$ and $\varphi_{i j} \cdot \varphi_{j k}=\varphi_{i k}$.

We claim that the presheaf $F$ satisfies the sheaf property. Indeed, let $v_{k} \in F\left(V_{k}\right)$ be a matching family for $F$ on a cover $\left(V_{k}\right)_{k \in K}$ of $V$. The $i$-th components of $v_{k}$ are a matching family for $F_{i}$ on the induced cover $\left(V_{k} \cap U_{i}\right)_{k \in K}$ of $V \cap U_{i}$ and the gluing operation $D\left(F_{i}\right)\left(V_{k} \cap\right.$ 
$\left.U_{i}\right) \rightarrow F_{i}\left(V \cap U_{i}\right)$ of a discrete stack is a bijection so that we obtain a glue $v \in F(V)$ of $v_{k}$ by gluing component-wise. This glue is unique because it is component-wise unique.

Let now $\omega_{i}: G\left|U_{i} \cong H\right| U_{i}, G, H \in \mathrm{U}(U)$ be a matching family of paths on a cover $\left(U_{i}\right)_{i \in I}$ of $U$. For $x \in G(V), V \subseteq U$ the family $\omega_{i} x$ in $H\left(V \cap U_{i}\right)$ is compatible because $\omega_{i}=\omega_{j}$ on $V \cap U_{i j}$. We define $\omega x$ to be the unique glue in $H(V)$ such that $(\omega x) \mid V \cap U_{i}=\omega_{i} x$. The uniqueness of glues allows us to verify that $\omega$ respects restriction and that the such defined $\omega$ is the unique path that agrees with $\omega_{i}$ on $U_{i}$.

We define $\mathrm{U} \vdash \mathrm{El}$ by taking El $F$ to be the small set $F(V)$ if $F$ is in $\mathrm{U}(V)$ and $\operatorname{El} \alpha\left(a, a^{\prime}\right)$ to be the set $\left\{0 \mid \alpha a=a^{\prime}\right\}$ if $\alpha$ is an isomorphism between $F$ and $F^{\prime}$ in $\mathrm{U}(V)$ and $a$ is in $F(V)$ and $a^{\prime}$ is in $F^{\prime}(V)$.

Theorem 4. The family $\mathrm{U} \vdash \mathrm{El}$ is a universal small and discrete stack: If $\Gamma \vdash A$ is small and discrete stack, there exists a unique map $|A|: \Gamma \rightarrow \mathrm{U}$ such that $\mathrm{El}|A|=A$ (with strict equality).

\subsection{Dependent sums}

Theorem 5. If $\Gamma \vdash A$ and $\Gamma$. $A \vdash B$ have stack structures then we can glue descent data and paths in $\Gamma \vdash \Sigma A B$.

Proof. Let $\left(\left(u_{i}, v_{i}\right),\left(\omega_{i j}, \mu_{i j}\right)\right) \in D(\Sigma A B) \rho$ be a descent datum on a covering $\left(U_{i}\right)_{i \in I}$ of $U$. We construct a glue $\left((u, v),\left(\omega_{i}, \mu_{i}\right)\right)$ that commutes with restriction.

Let $\left(u, \omega_{i}\right)$ to be the glue of the datum $\left(u_{i}, \omega_{i j}\right) \in D(A) \rho$. We describe a descent datum in $D(B)(\rho, a)$. The object part of this descent datum is given by $v_{i} \omega_{i}^{-1} \in B\left(\rho\left|U_{i}, u\right| U_{i}\right)$. We have then paths $\left(v_{i} \omega_{i}^{-1} \uparrow \omega_{i}\right): v_{i} \omega_{i}^{-1} \cong \omega_{i} v_{i}$ and thus paths

$$
\left(v_{i} \omega_{i}^{-1} \uparrow \omega_{i}\right)\left|U_{i j} \cdot \mu_{i j} \cdot\left(v_{j} \omega_{j}^{-1} \uparrow \omega_{j}\right)^{-1}\right| U_{i j}: v_{i} \omega_{i}^{-1}\left|U_{i j} \cong v_{j} \omega_{j}^{-1}\right| U_{i j}
$$

These satisfy the cocycle condition. Thus we have a descent datum in $D(B)(\rho, u)$. Let $\left(v, \mu_{i}^{\prime}\right)$ be the glue of this datum. We have paths $\mu_{i}:=\mu_{i}^{\prime} \cdot\left(v_{i} \omega_{i}^{-1} \uparrow \omega_{i}\right): v \mid U_{i} \cong \omega_{i} v_{i}$

We then take the glue of $\left(\left(u_{i}, v_{i}\right),\left(\omega_{i j}, \mu_{i j}\right)\right)$ to be given by $\left((u, v),\left(\omega_{i}, \mu_{i}\right)\right)$. Since

$$
\mu_{i}^{\prime}\left|U_{i j} \cdot\left(v_{i} \omega_{i}^{-1} \uparrow \omega_{i}\right)\right| U_{i j} \cdot \mu_{i j} \cdot\left(v_{j} \omega_{j}^{-1} \uparrow \omega_{j}\right)^{-1}\left|U_{i j}=\mu_{j}^{\prime}\right| U_{i j}
$$

we have that $\mu_{i}\left|U_{i j} \cdot \mu_{i j}=\mu_{j}\right| U_{i j}$.

Let $\alpha: \rho \cong \rho^{\prime}$. Given a matching family of paths $\left(\omega_{i}, \mu_{i}\right):(u, v) \mid U_{i} \cong_{\alpha \mid U_{i}}\left(u^{\prime}, v^{\prime}\right)$. Since $A$ have a stack structure we have a unique $\omega: u \cong_{\alpha} u^{\prime}$ with $\omega \mid U_{i}=\omega_{i}$. But then $\mu_{i}: v\left|U_{i} \cong(\alpha, \omega)\right| U_{i}$ $v^{\prime} \mid U_{i}$ is a matching family for the stack $B$ and thus have a unique $\mu: v \cong_{(\alpha, \omega)} v^{\prime}$ where $\mu \mid U_{i}=\mu_{i}$.

\subsection{Paths}

Descent data for the discrete family $\Gamma \vdash$ Path $A a_{0} a_{1}$ correspond to matching families of paths for $A$ and they have unique glues if $A$ is a prestack. Unique choice then gives us a function from descent data to glues for Path $A a_{0} a_{1}$ which, also by uniqueness, necessarily commutes with restriction.

Proposition 4. If $\Gamma \vdash A$ has a stack structure and $\Gamma \vdash a_{0}: A$ and $\Gamma \vdash a_{1}: A$ then $\Gamma \vdash$ Path $A a_{0} a_{1}$ has a discrete stack structure. 


\subsection{Univalence}

An equivalence between two types $A$ and $B$ is a map $f: A \rightarrow B$ such that for each $y: B$ the fiber of $f$ above $y$ is contractible. If both $A$ and $B$ are discrete stacks, then $f$ being contractible means that $f$ is an isomorphism. As in [9, 5.4], we have then a one-to-one correspondence between the type of equivalences $\mathrm{El} a \simeq \mathrm{El} b$ and the type of paths Path $\mathrm{U} a b$.

\subsection{Propositional truncation}

We define the family of sets $\|A\| \rho$ inductively. For every basic open $U, \rho \in \Gamma(U)$ and $u \in A \rho$ let $u \in\|A\| \rho$. Moreover, for every covering $\left(U_{i}\right)_{i \in I}$ of a given basic open $U, \rho \in \Gamma(U)$ and $u_{i} \in\|A\|\left(\rho \mid U_{i}\right)$ let $\left(U_{i}, u_{i}\right)_{i \in I} \in\|A\| \rho$. Notice that this forms a set since the index set $I$ is restricted to be small. (Without this restriction, we will get a class and not a set in general.) Then, we define the family of functions $\|A\| \rho \rightarrow\|A\|(\rho \mid V)$ recursively. For every pair of basic opens $V \subseteq U, \rho \in \Gamma(U)$ and $x \in\|A\| \rho$ let $x|V:=u| V$ if $x=u$ with $u \in A \rho$ and $x \mid V:=\left(U_{j} \cap V, u_{j} \mid U_{j} \cap V\right)_{j \in J}$, where $J \subseteq I$ is the restriction to indices $i \in I$ such that $U_{i}$ meets $V$, if $x=\left(U_{i}, u_{i}\right)_{i \in I}$ with $u_{i} \in\|A\|\left(\rho \mid U_{i}\right)$. Lastly, we define the type family $\|A\|$ to be the proposition on the family of sets and functions just defined. The collection of discrete families $\Gamma \vdash A$ discrete is not closed under propositional truncation: Given $\alpha: \rho \cong \rho^{\prime}$ and $x \in\|A\| \rho$, then there is a unique path $x \cong_{\alpha} x^{\prime}$ that connects $x$ to each element $x^{\prime} \in\|A\| \rho^{\prime}$.

The family $\Gamma \vdash\|A\|$ always has a stack structure, even without assuming one on $\Gamma \vdash A$. If we have a covering $\left(V_{l}\right)_{l \in L}$ of $U$ and for each $l$ in $L$ we have an element $x_{l}$ of $\|A\|\left(\rho \mid V_{l}\right)$, then this family $x_{l}$ always defines in a unique way a descent datum and we can consider the family $\left(V_{l}, x_{l}\right)_{l \in L}$, which defines a gluing of the family $x_{l}$. This operation furthermore satisfies the functoriality condition.

\subsection{Example: One-point space}

One of the simplest examples of the notion of stack is that where the poset of basic opens has exactly one object $V$. In that case, a covering of $V$ is a nonempty finite family $\left(V_{i}\right)_{i \in I}$ where each $V_{i}=V$ and a stack is a single groupoid $G$ with a gluing operation. That is, for any family $\left(u_{i}\right)_{i \in I}$ of elements in $G$ and paths $\varphi_{i j}: u_{i} \cong u_{j}$ satisfying $\varphi_{i j} \cdot \varphi_{j k}=\varphi_{i k}$ we have glue $\left(u_{i}, \varphi_{i j}\right)=\left(u, \varphi_{i}\right)$ such that $\varphi_{i}: u \cong u_{i}$ and $\varphi_{i} \cdot \varphi_{i j}=\varphi_{j}$. We can use this example to motivate the definition of propositional truncation given above. Suppose we naively truncate the groupoid $G$ to get the proposition $\|G\|$ on the objects of $G$, then we have no way of defining the gluing operation on $\|G\|$ since we lack a particular choice of glue for a given descent datum.

\section{Countable choice}

\subsection{A stack model where countable choice does not hold}

We write $U, V, W, \ldots$ nonempty open rational intervals included in the open unit interval $(0,1)$. For each $n$, and $i=1, \ldots, n$ we let $U_{i}^{n}$ be $((i-1) /(n+1),(i+1) /(n+1))$ so that $\left(U_{i}^{n}\right)_{i=1, \ldots, n}$ is a covering of $(0,1)$.

We let $|\mathrm{N}|$ be the constant presheaf where each $|\mathrm{N}|(V)$ is the set $\mathbb{N}$ of natural numbers and $\mathrm{N}=\mathrm{El}|\mathrm{N}|$. We have [15]

Lemma 3. $|\mathrm{N}|$ is a (small) sheaf.

It is also well-known that in the sheaf model over $(0,1)$, there are Dedekind reals that are not Cauchy reals [15]. It is simple to transform this fact to a counter-example to our type-theoretic version of countable choice. 
We define $A: \mathrm{N} \rightarrow \mathrm{U}$ by letting $A n$ be the subsheaf of the (small) constant sheaf $|\mathrm{Q}|(V)=\mathbb{Q}$ of rational numbers

$$
(A n)(V)=\left\{r \in \mathbb{Q}|\forall(x \in V)| x-r \mid<\frac{1}{n+1}\right\}
$$

Notice that each $i /(n+1)$ is an element of $(A n)\left(U_{i}^{n}\right)$.

Proposition 5. In this model

1. the type $\Pi(n: \mathrm{N}) \| \mathrm{EI}($ An $n) \|$ is inhabited

2. the type $\Pi(n: \mathrm{N}) \mathrm{El}(A n)$, and hence also the type $\|\Pi(n: \mathrm{N}) \mathrm{El}(A n)\|$, is empty

Proof. For each open set $V$, we let $s_{V} n$ be the family $\left(V \cap U_{i}^{n}, i /(n+1)\right), i$ such that $V$ and $U_{i}^{n}$ meet, in $\|\mathrm{El}(A n)\|(V)$. Since we have $\left(s_{V} n\right) \mid W=s_{W} n$ if $W \subseteq V$, this defines a section of $\Pi(n: \mathrm{N})\|\mathrm{El}(A n)\|$.

For the second point, it is enough to notice that, for each given $V$, the set

$$
(A n)(V)=\left\{r \in \mathbb{Q}|\forall(x \in V)| x-r \mid<\frac{1}{n+1}\right\}
$$

is empty for $n$ large enough.

Corollary 1. In this model, the principle of countable choice CC does not hold.

Corollary 2. One cannot show countable choice in type theory with one univalent universe and propositional truncation.

\section{Markov's principle}

The interpretation of the type $\mathbf{N}$ was especially simple on the space $(0,1)$ using the fact that its basic opens are connected. We will now consider the "dual" case where the space is totally disconnected. We assume from now on that the basic opens are nonzero elements $e, e^{\prime}, \ldots$ of a Boolean algebra with decidable equality. We consider only coverings of $e$ given by a finite partition $e_{i}, i \in I$, of $e$, that is a finite set of disjoint elements $e_{i} \leqslant e$ such that $e=\bigvee_{i \in I} e_{i}$.

Given a type family $\Gamma \vdash A$ and $\rho \in \Gamma(e)$, a descent datum $d \in D(A) \rho$ for this family is now simply given by a partition $e_{i}, i \in I$, of $e$ and a family $u_{i} \in A \rho \mid e_{i}$.

We can now strengthen the notion of stack structure by further imposing that we have (glue $d) \mid e_{i}=u_{i}$ for $d=\left(u_{i}\right) \in D(A) \rho$. This strict gluing condition states that the required equalities between (glue $d) \mid e_{i}$ and $u_{i}$ are strict equalities. In fact, it is enough to require glue $(u)=u$ for partitions consisting of exactly one element.

This refinement is needed for the elimination of natural numbers and Booleans in the universe.

Proposition 6. If $\Gamma . A \vdash B$ satisfies the strict gluing condition, then so does $\Gamma \vdash \Pi A B$.

Proposition 7. If $\Gamma \vdash A$ and $\Gamma$. $A \vdash B$ satisfy the strict gluing condition, then so does $\Gamma \vdash \Sigma A B$.

If $\mathrm{U}(e)$ is the collection of sheaves on $e$, we can refine the stack structure on $\mathrm{U}$ in order to satisfy the strict gluing condition: If $e_{i}$ is a partition of $e$ and $F_{i}$ is a sheaf on $e_{i}$ we define $F=\operatorname{glue}\left(e_{i}, F_{i}\right)$ by taking $F\left(e^{\prime}\right)$, for $e^{\prime} \leqslant e$, to be the product of all $F_{i}\left(e^{\prime} \wedge e_{i}\right)$ if $e^{\prime}$ meets strictly more than one $e_{i}$, and to be exactly $F_{i}\left(e^{\prime}\right)$ if $e^{\prime} \leqslant e_{i}$. This defines a sheaf, and the functoriality law glue $\left(e_{i}, F_{i}\right) \mid e^{\prime}=\operatorname{glue}\left(e_{i} \wedge e^{\prime}, F_{i} \mid e_{i} \wedge e^{\prime}\right)$ is satisfied. 


\subsection{Natural numbers and Booleans}

We define the sheaf $|\mathrm{N}|$ by taking $|\mathrm{N}|(e)$ to be the set of families $\left(e_{i}, n_{i}\right)$ where $e_{i}$ is a partition of $e$ and $n_{i} \neq n_{j}$ if $i \neq j$. We define similarly $\left|\mathrm{N}_{2}\right|$ where $n_{i}$ can only take the values 0 or 1 , and $\left|N_{1}\right|(e)=\{0\}$, and $\left|N_{0}\right|(e)$ is the empty set. We define then $N=E I|N|$ and similarly for $N_{2}, N_{1}$ and $\mathrm{N}_{0}$.

We define $\operatorname{suc}\left(e_{i}, n_{i}\right)$ to be $\left(e_{i}, n_{i}+1\right)$ and $0(e)$ is the element $(e, 0)$.

The rec operator is then defined as a section of $\Gamma . \mathrm{N} \vdash C$

$$
\begin{aligned}
& (\operatorname{rec} c d)(\rho, 0) \quad=c \rho \\
& (\operatorname{rec} c d)(\rho, n+1)=d(\rho, n,(\operatorname{rec} c d)(\rho, n)), \text { where } n \in \mathbb{N} \\
& (\operatorname{rec} c d)\left(\rho,\left(e_{i}, n_{i}\right)\right)=\operatorname{glue}\left(e_{i},(\operatorname{rec} c d)\left(\rho \mid e_{i}, n_{i}\right)\right)
\end{aligned}
$$

given sections $\Gamma \vdash c: C[0]$ and $\Gamma$. N. $C \vdash d: C[\operatorname{suc} \mathbf{q}] \mathbf{p}$.

We remark that the strict gluing condition is needed to make the above definition work, i.e. so that for $m \in \mathrm{N}(e)$ and $e^{\prime} \leqslant e$ we have $((\operatorname{rec} c d) \rho m)\left|e^{\prime}=(\operatorname{rec} c d) \rho\right| e^{\prime} m \mid e^{\prime}$.

\subsection{A stack model where Markov's principle does not hold}

We can express Markov's principle in type theory by the type:

$$
\mathrm{MP}:=\Pi\left(h: \mathrm{N} \rightarrow \mathrm{N}_{2}\right)(\neg \neg(\Sigma(x: \mathrm{N}) \text { El isZero }(h x)) \rightarrow \Sigma(x: \mathrm{N}) \text { El isZero }(h x))
$$

where isZero: $\mathrm{N}_{2} \rightarrow \mathrm{U}$ is defined by isZero:= $\lambda y \cdot \operatorname{rec}_{2}\left|\mathrm{~N}_{1}\right|\left|\mathrm{N}_{0}\right| y$ and the type $\neg A$ by $A \rightarrow \mathrm{N}_{0}$.

We could also consider the version where we use weak existential $\exists(x: A) B=\|\Sigma(x: A) B\|$ instead of sigma type, but the two versions are logically equivalent [16, Exercise 3.19].

Take a countably infinite set of variables $p_{0}, p_{1}, \ldots$ Consider the free Boolean algebra generated by the atomic formulae $p_{n}$. We write $p_{n}=0$ for $\neg p_{n}$ and $p_{n}=1$ for $p_{n}$. An object $e$ in this algebra represents then a compact open in Cantor space $\{0,1\}^{\mathbb{N}}$, where a conjunctive formula $\bigwedge p_{i}=b_{i}$ represents the set of sequences in $\{0,1\}^{\mathbb{N}}$ having value $b_{i}$ at index $i$. A formula $e$ in the algebra is then a finite disjunction of these.

We have an interpretation of type theory in stacks over this algebra, and we are going to see that Markov's principle is not valid in this interpretation. We define $\mathrm{f}$ in $\mathrm{N} \rightarrow \mathrm{N}_{2}$ by taking $\mathrm{f} n$, $n \in \mathrm{N}(e)$, at $e_{i}$ to be $\left(\left(e_{0}, 0\right),\left(e_{1}, 1\right)\right)$ where $e_{b}$ is $e_{i} \wedge\left(p_{n_{i}}=b\right)$ if $e_{i}$ meets both $\left(p_{n_{i}}=0\right)$ and $\left(p_{n_{i}}=1\right)$, and to be $\left(e_{i}, b\right)$ if $e_{i} \leqslant\left(p_{n_{i}}=b\right)$.

Proposition 8. In this model

1. $\neg \neg(\Sigma(x: \mathrm{N})$ El isZero $(\mathrm{f} x))$ is inhabited.

2. $\Sigma(x: \mathrm{N})$ El isZero $(\mathrm{f} x)$ is not inhabited.

Proof. To show that $\neg \neg(\Sigma(x: \mathrm{N})$ El isZero $(\mathrm{f} x))$ is inhabited it is sufficient to show that for all $e$ the set $(\neg(\Sigma(x: \mathrm{N})$ El isZero $(\mathrm{f} x)))(e)$ is empty. For that it will be sufficient to show that for some $e^{\prime} \leqslant e$ we have that $(\Sigma(x: \mathrm{N}) \operatorname{El}$ isZero $(\mathrm{f} x))\left(e^{\prime}\right)$ is not empty. But given any $e$ we can simply choose $e^{\prime}=\left(p_{n}=0\right) \wedge e$ for some $n$ big enough. Thus El isZero $(\mathrm{f} n)$ at $e^{\prime}$ is $\{0\}$ and $(\Sigma(x: \mathrm{N})$ El isZero $(\mathrm{f} x))\left(e^{\prime}\right)$ is not empty.

We now show that $\Sigma(x: \mathrm{N})$ El isZero $(\mathrm{f} x)$ is not inhabited. For any $n=\left(e_{i}, n_{i}\right)$ in $\mathrm{N}(1)$ where $\left(e_{i}\right)$ is a partition of 1 , we can find exactly one $e_{i}$ which contains (as a compact open subset of Cantor space) the constant function 1 . This element $e_{i}$ meets $p_{n_{i}}=1$ so that El isZero $(\mathrm{f} n)$ is the empty set at $\left(p_{n_{i}}=1\right) \wedge e_{i}$ and hence also at 1 .

Corollary 3. In this model Markov's principle does not hold. 
Corollary 4. One cannot show Markov's principle in type theory with one univalent universe.

The situation however is different from the one of countable choice. The following provides an alternative argument that Markov's principle cannot be proved in type theory with one univalent universe ${ }^{3}$.

Proposition 9. Markov's principle does not hold in the groupoid model in a set theory where Markov's principle does not hold (for instance in suitable sheaf models of CZF [6]).

In 4 it was shown that Markov's principle is independent from type theory with one (nonunivalent) universe. The paper describes an extension of type theory where the principle does not hold and proves the consistency of that extension with a normalization argument. We note however that the model given here does not give an interpretation of the extended type theory in [4]. In particular the universe (inductively defined) in that extension satisfies the sheaf property.

\section{Conclusion}

One special case of sheaf models are Boolean-valued models, for instance as in the work [14], and it would be interesting to formulate a stack version of these models as well.

We expect that essentially the same kind of models can be defined over a site and not only over a topological space. In particular, it should be possible to extend the sheaf model in [11] to a stack model of type theory with an algebraic closure of a given field, where existence of roots is formulated using propositional truncation (as explained in the cited work, this existence cannot be stated using strong existence expressed by sigma types). Another example could be a stack version of Schanuel topos used in the theory of nominal sets [12].

As stated in the introduction, the argument should generalize to an $\infty$-stack version of the cubical set model [3]. The coherence condition on descent data will be infinitary in general, but it will become finitary when we restrict the homotopy level (and empty in particular in the case of propositions).

\section{References}

[1] Marc Bezem, Thierry Coquand, and Simon Huber. A model of type theory in cubical sets. In Ralph Matthes and Aleksy Schubert, editors, 19th International Conference on Types for Proofs and Programs (TYPES 2013), volume 26 of Leibniz International Proceedings in Informatics (LIPIcs), pages 107-128, Dagstuhl, Germany, 2014. Schloss Dagstuhl-LeibnizZentrum fuer Informatik.

[2] Alonzo Church. A formulation of the simple theory of types. The Journal of Symbolic Logic, 5(2):56-68, 1940.

[3] Cyril Cohen, Thierry Coquand, Simon Huber, and Anders Mörtberg. Cubical type theory: a constructive interpretation of the univalence axiom. CoRR, abs/1611.02108, 2016.

[4] Thierry Coquand and Bassel Mannaa. The independence of Markov's principle in type theory. In Delia Kesner and Brigitte Pientka, editors, 1st International Conference on Formal Structures for Computation and Deduction, FSCD 2016, June 22-26, 2016, Porto, Portugal, volume 52 of LIPIcs, pages 17:1-17:18. Schloss Dagstuhl - Leibniz-Zentrum fuer Informatik, 2016.

\footnotetext{
${ }^{3}$ This argument gives also a proof that Markov's principle is independent of a hierarchy of univalent universes by considering the cubical set model [3] in a set theory where Markov's principle does not hold.
} 
[5] Laura Crosilla and Michael Rathjen. Inaccessible set axioms may have little consistency strength. Annals of Pure and Applied Logic, 115(1-3):33-70, 2002.

[6] Nicola Gambino. Heyting-valued interpretations for constructive set theory. Annals of Pure and Applied Logic, 137(1-3):164-188, 2006.

[7] Jean Giraud. Cohomologie non abélienne. Springer-Verlag, Berlin-New York, 1971. Die Grundlehren der mathematischen Wissenschaften, Band 179.

[8] Alexander Grothendieck and Jean Dieudonné. Éléments de géométrie algébrique. I. Le langage des schémas. Institut des Hautes Études Scientifiques. Publications Mathématiques, (4):228, 1960

[9] Martin Hofmann and Thomas Streicher. The groupoid interpretation of type theory. In Twenty-five years of constructive type theory (Venice, 1995), volume 36 of Oxford Logic Guides, pages 83-111. Oxford Univ. Press, New York, 1998.

[10] Martin Hofmann and Thomas Streicher. Lifting Grothendieck universes. December 2014.

[11] Bassel Mannaa and Thierry Coquand. A sheaf model of the algebraic closure. In Paulo Oliva, editor, Proceedings Fifth International Workshop on Classical Logic and Computation, CLEC 2014, Vienna, Austria, July 13, 2014., volume 164 of EPTCS, pages 18-32, 2014 .

[12] Andrew M. Pitts. Nominal Sets: Names and Symmetry in Computer Science, volume 57 of Cambridge Tracts in Theoretical Computer Science. Cambridge University Press, 2013.

[13] Fabian Ruch. The path model of intensional type theory. Master's thesis, Chalmers University of Technology, Gothenburg, Sweden, 2015.

[14] Dana S. Scott. Stochastic $\lambda$-calculi: an extended abstract. Journal of Applied Logic, 12(3):369-376, 2014.

[15] Anne S. Troelstra and Dirk van Dalen. Constructivism in Mathematics: An Introduction, volume II of Studies in Logic and the Foundations of Mathematics. North-Holland, 1988.

[16] The Univalent Foundations Program. Homotopy Type Theory: Univalent Foundations of Mathematics. http://homotopytypetheory.org/book, Institute for Advanced Study, 2013.

[17] Vladimir Voevodsky. The equivalence axiom and univalent models of type theory. (Talk at CMU on February 4, 2010). ArXiv e-prints, February 2014.

[18] Chuangjie Xu and Martín Escardó. Universes in sheaf models. February 2016. 